\title{
АНАЛИЗ СИСТЕМ ТРУБОПРОВОДНОГО ТРАНСПОРТА САХАРНЫХ ЗАВОДОВ С ЭЛЕМЕНТАМИ АВТОМАТИЧЕСКОГО РЕГУЛИРОВАНИЯ
}

Analysis of systems of transport valves of sugar-houses with elements of automatic control

\author{
Володин С.А. ${ }^{1}$, Мирончук В.Г., ${ }^{2}$ Кривопляс-Володина Л.А. ${ }^{2}$ (Volodyn S.A., Myronchuk V.G., Kryvoplyas- \\ Volodyna L.A.) \\ ${ }^{1} \mathrm{OOО} «$ КАМОЦЦИ», \\ ${ }^{2}$ Национальный университет пищевых технологий, Киев \\ ${ }^{2}$ ORCID: 0000-0003-1516-6153
}

Copyright (C) 2014 by author and the journal "Automation technological and business - processes". This work is licensed under the Creative Commons Attribution International License (CC BY). http://creativecommons.org/licenses/by/4.0/

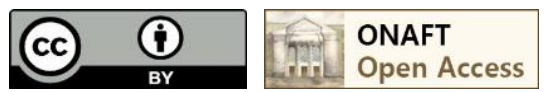

\section{DOI: $10.15673 /$}

\section{Аннотация}

Повышенные требования к качеству сахара и снижения его себестоимости, без больших капитальных вложений, могут быть обеспечены внедрением систем автоматизации технологических станций сахарного производства и созданием единой системы управления потоками сахарного производства и его энергозатратами.На современном производстве сахара из свеклы, при классической технологии его получения, должны присутствовать такие этапы переработки свеклы: подача, мойка, очистка, диффузия, дефекосатурация, выпарка, кристаллизация, сушка и упаковка. Получение максимального выхода сахара зависит от наличия соответствующего оборудования и трубопроводов, которые соединяют это оборудование. Большинство сахарных заводов работают по непрерывному циклу получения сахара, то есть подача свеклы, переработка, получение сока, очистка, выпаривание и кристаллизация осуществляются без остановок в технологическом процессе работы завода. Оборудование, которое используется при получении сахара, имеет свои технические параметры, соответствующие определенной производительности. Кроме технологического оборудования с соответствующей производительностью, необходимо иметь и соответствующие трубопроводные соединения между этим оборудованием, которые позволят пропускать перерабатываемую продукцию[4]. Решение этой проблемы тесно связано с обоснованным выбором компоновки трубопроводной и запорно-регулирующей системы, например: паровыми, водяными, конденсатными, соковыми, сиропными и паточными, а также трубопроводами для подачи извести и известкового молока. Постоянная эксплуатация трубопроводов приводит к образованию на внутренних стенках всевозможных отложений и накипи. Кроме того, сечения трубопроводов не всегда правильно подобраны под необходмую производительность завода. Поиск решения в механическом изменении сечений труб не дает возможности точно и оперативно влиять на технологический процесс. Поэтому в статье проведен анализ выбора и использования средств автоматизации, а также современных систем управления технологическими процессами, с примененим трубопроводной арматуры на базе электропневматических позиционеров «Камоцци» с реализацией обратной связи с помощью бесконтактных пропорциональных датчиков положения с токовым выходом. Рассмотренные решения позволяют не только четко необходимые режимы работы, но и изменять принятые решения при резких отклонениях в работе системы управления[1-4]. Получены результаты по определению оптимальных значений сечений 
трубопроводов на разных технологических уастках сахарных заводов, необходимых для поддержания технологических режимных параметров продукции (сок, сироп, известковое молоко и т.д.), расхода пара и воды. Приведенные результаты исследования дают возможность использовать в системе управления запорной арматурой электропневмопозиционеры.

\section{Absctract}

For upgrading productions of sugar and decline of his prime price, without large capital investments, it is necessary to provide introduction of the systems of automation of the technological stations of saccharine production. And also creation of single control system by the streams of saccharine production and his energy consumptions. On the modern production of sugar from a beet, at classic technology of his production, such stages of processing of beet must be present as: serve, wash, clearing, diffusion, defecosaturation, boil-off, crystallization, drainage and packing. The receipt of maximal rendement depends on the presence of corresponding equipment and pipelines that connect this equipment. Most sugar-houses work on the continuous cycle of receipt of sugar, id est the serve of beet, processing, receipt of juice, cleaning, evaporation and crystallization, come true without stopping at the technological process of work of plant. An equipment that is used for the receipt of sugar has the technical parameters corresponding to the certain productivity. Except a technological equipment with the corresponding productivity, it is necessary to have corresponding pipeline connections between this equipment, that will allow to skip the processed products. The decision of this problem is closely related to the reasonable decision for arrangement of the pipeline and systems of transport valves, for example for: steam, aquatic, runback, exit-juice, syrup and molasses, and also by pipes for a lime and limewater. Permanent exploitation of pipelines results in education on the midwalls of various sedimentations and scum. In addition, the sections of pipelines not always are correctly neat under the necessary productivity of plant. The search of decision in the mechanical change of sections of pipes does not give an opportunity exactly and operatively to influence on a technological process. The analysis of choice and use of facilities of automation is conducted in the article, and also modern control system by technological processes, with примененим of pipeline armature on the base of electro-pneumatic позиционеров оf "Камоцци" with realization of feed-back by means of contactless proportional pick-ups of position with a current exit. The considered decisions allow not only clearly to support necessary office hours but also to change made decision at sharp rejections in-process control system. Results are got on determination of optimal values of sections of pipelines on the different technological areas of sugar-houses, necessary for maintenance of technological regime parameters of products (juice, syrup, limewater etc.), expense of steam and water. Research results give an opportunity to use, positioning of actuators for control.

Ключевые слова

Трубопроводная арматура, позиционный привод, погрешность, обратная связь.

Введение

Диффузия, дефекосатурация, выпарка и кристаллизация являются необходимыми этапами в технологическом процессе получения сахара, но для экономии и оптимизации тепловых энергетических затрат наиболее важным является выпарное отделение. Для того чтобы необходимое количество пара и жидкостей с соответствующими технологическим требованиям температурой и давлением поступало к потребителям, необходимо осуществлять регулирование сечений прохождения жидкостей и пара за счет применения различного вида запорных и регулирующих устройств [5].

Используемые на сахарных заводах трубопроводы и запорные устройства имеют стандартные диаметры и соответствующие сечения труб. В случае несоответствия сечения воздушного и парового трубопроводов расчетным параметрам увеличиваются шумы, то есть нарушается режим работы. Для выбора запорных и регулирующих устройств могут быть использованы материалы каталога компании ООО «Камоцци»[1], которая профессионально занимается созданием комплексных решений по обеспечению работы технологических процессов в разных отраслях, в т.ч. и для пищевой. В каталоге приведены серийно выпускаемые и наиболее часто применяемые конструкции трубопроводной арматуры. Но при введении подобранного устройства в технологический процес, необходимо предварительно проверить обеспечение его расходных характеристик на заданном технологическом участке. 
Анализ существующих решений

Отсутствие алгоритма правильного подбора трубопроводов по основным рациональным критериям на сахарных заводах, - является важным недостатком общей компоновки технологического процесса, который может нарушить общую технологическую продуктивность. Так, например, вместо двух трубопроводов диаметром 100мм ставится общий трубопровод диаметром 200мм, но может быть и наоборот, когда вместо одного трубопровода диаметром 200мм ставятся два диаметром 100мм. Полученная компоновка трубопровода приводит к резкому возрастанию шумов на заводе и нарушению распределения пара по заводу, уменьшению вакуума в вакуумаппаратах и т.д.

Реальный технологический процесс сопровождается постоянным изменением расходов сырья, растворов, необходимости текущего изменения пропускной способности трубопроводов для обеспечения оптимальных условий протекания химических тепловых преобразований. Таким образом, возникает необходимость автоматического регулирования сечений трубопроводов путем использования заслонок, клапанов, кранов, имеющих в качестве привода пневматические и электропневматические системы слежения.

Выбор диаметров трубопроводов (с учетом их сечения) влияет на скорость и режимы движения технологической среды. Техническая производительность трубопроводов зависит от производительности завода [3-4]:

$$
A=\frac{T \cdot \pi \cdot 100 \cdot D^{2} \cdot u \cdot \rho}{4 \cdot k \cdot a}, \text { (т/сут) }
$$

где Т - продолжительность суток, (c); u - скорость движения продуктов в трубопроводе, м/с; $\rho$ - плотность перемещаемого продукта, т/м³ ; а - количество перемещаемого продукта, \% к массе свеклы; $\mathrm{k}$ - коэффициент неравномерности поступления среды; (например, для трубопроводов подвода пара к выпарной станции $\mathrm{k}=1,5 . .1,6$; остальных корпусов выпарной установки $\mathrm{k}=1,25$; для сока, сиропа, известкового молока, воды $\mathrm{k}=1,0$...1,5. [4]

Искомая величина D (диаметр трубопровода) определяется по заданной необходимой мощности завода:

$$
D=\sqrt{\frac{A \cdot 4 \cdot k \cdot a}{T \cdot \pi \cdot 100 \cdot u \cdot \rho}}, \text { (м) }
$$

Учитывая анализ работы трубопроводов на сахарных предприятиях Украины, проведен расчет по определению необходимых сечений труб для подачи сока и пара на отдельных участках. Например для выпарного отделения диаметры труб составили для движения сока - 0,159 м, для сиропа - 0,100 м.

Таким образом, учитывая условия технологического процеса можно подобрать оптимальные сечения трубопроводов.

\section{Постановка задачи}

Влияние отсечных клапанов, кранов, заслонок на условия работы трубопроводного транспорта в целом, с учетом подобранных выше конструктивных параметров, неразделимо с решением вопросов, связанных с управлением запорной арматурой (выбор силовой (пневматической) части привода, системы управления и т. д. Эксплуатационные свойства исполнительных устройств (регулирующих клапанов) в значительной мере определяют основные характеристики: гидравлические, силовые и конструктивные для привода в целом. Учитывая характеристики исполнительных устройств, такие как: пропускная способность $K_{v}$ (определяется объемным расходом среды в $\left(\mathrm{M}^{3} / 4\right)$ ), плотность замыкания (постепенно изменяемая регулирующим органом при перепаде давления на приводе в $0,1 \mathrm{MПа} \mathrm{-} \mathrm{можно} \mathrm{рассчитывать} \mathrm{текущее} \mathrm{значение} \mathrm{пропускной} \mathrm{способности} \mathrm{при} \mathrm{заданной}$ величине хода рабочего звена (штока пневмоцилиндра) в процентах.

По диапазону изменения пропускной способности запорно-регулирующей арматуры, можно описать пропускную характеристику $K_{v}=f(S)$, определять зависимость пропускной способности от перемещения затвора $\mathrm{S}$, связанного с рабочим звеном привода (штоком пневмоцилиндра). При линейной пропускной характеристике обеспечивается пропорциональная зависимость между пропускной способностью клапана и ходом штока. При равно процентной пропускной характеристике обеспечивается приращение пропускной способности клапана пропорционально текущему значению пропускной способности по ходу, т. е. чем больше ход, тем больше увеличивается $K_{v}$ на единицу хода. Линейная и равно процентная пропускные характеристики - эти зависимости действительны при постоянном перепаде давлений на клапане, т. е. в условиях, когда перепад давлений на клапане не зависит от расхода среды, а в регулируемой системе все остальные гидравлические сопротивления по сравнению с гидравлическим сопротивлением клапана пренебрежимо малы. В таких условиях расходная 
характеристика клапана совпадает с его пропускной характеристикой[5-7]. Зависимость пропускной способности трубопровода от хода рабочего звена(плунжера) в отсечном клапане, при постоянном перепаде давлений на участке, определяет собой пропускную характеристику регулирующего клапана. Она зависит от профиля плунжера. Наиболее часто применяются линейная и равнопроцентная пропускные характеристики. При линейной пропускной характеристике обеспечивается пропорциональная зависимость между пропускной способностью клапана и ходом плунжера.
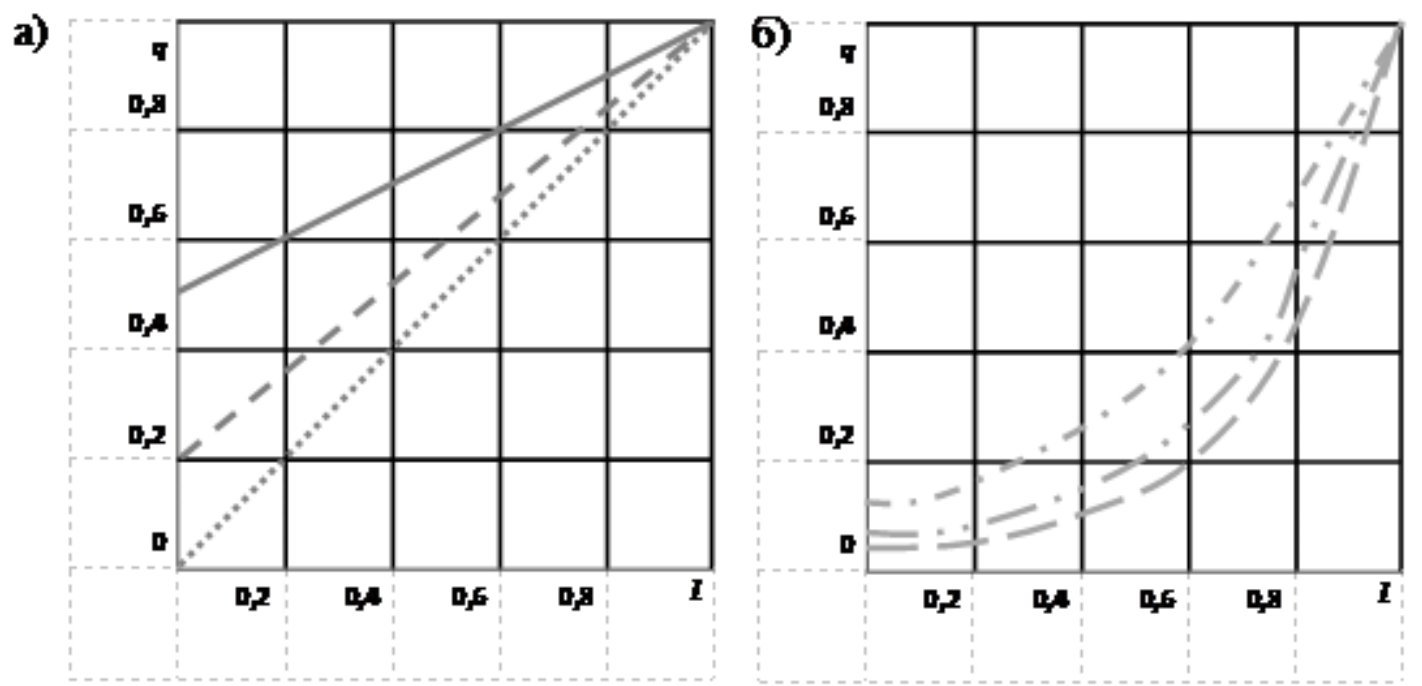

Рис.1 - Пропускные характеристики $q=f(l)$ регулирующих клапанов: а) линейные; б) равнопроцентные

При равнопроцентной пропускной характеристике обеспечивается приращение пропускной способности клапана пропорционально текущему значению пропускной способности по ходу клапана, т. е. чем больше ход клапана, тем больше увеличивается $K_{v}$ на единицу хода. Для удобства анализа, оценки и расчетов пропускные и расходные характеристики могут выражаться в относительных (безразмерных) величинах. При этом $q=K_{v} / K_{v}$ - относительный расход среды, изменяющийся от 0 до $1 ; S=S / S_{y}$ - относительный ход плунжера, изменяющийся от 0 до 1. Условная пропускная способность $K_{v}$ представляет собой номинальное значение величины пропускной способности при условном ходе затвора, выраженное в (м3/ч). Таким образом, $q=f(l)-$ пропускная характеристика клапана в относительных величинах, а $K_{v}=f(S)$ - в абсолютных. Использование пропускных характеристик в безразмерном виде удобно для общей оценки различных по размерам клапанов.

При малых значениях $K_{v}$, пропускная способность может не соответствовать пропускной характеристике. Отношение условного значения пропускной способности клапана к наименьшей в пределах пропускной характеристики представляет собой диапазон изменения пропускной характеристики, который в серийных двухседельных клапанах при линейной характеристике равен 7.5, а при равнопроцентной — 24.

На рис.1 изображены линейная и равнопроцентная пропускные характеристики. Эти зависимости действительны при постоянном перепаде давлений на клапане, т. е. в условиях, когда перепад давлений на клапане не зависит от расхода среды, а в регулируемой системе все остальные гидравлические сопротивления по сравнению с гидравлическим сопротивлением клапана пренебрежимо малы. В таких условиях расходная характеристика клапана совпадает с его пропускной характеристикой.

В реальных условиях эксплуатации трубопроводных систем, перепад давлений на регулирующем клапане не остается постоянным, а изменяется в зависимости от гидравлических характеристик насосной установки, составляющих элементов трубопроводной системы, расхода среды потребителями, свойств перемещаемой среды, ее вязкости, гидравлического режима движения, способности вскипания в связи с понижением давления и некоторых других факторов.

Расчетный участок трубопровода (системы) с давлением $\mathrm{P}_{0}$ в начале и $\mathrm{P}_{\mathrm{n}}$ в конце участка можно представить как состоящий из линии, включающей трубопровод и технологические аппараты, в которой потери давления 
составляют ${ }_{\Delta} \mathrm{P}$. Таким образом, ${ }_{\Delta} \mathrm{P}={ }_{\Delta} \mathrm{P}_{\mathrm{c}}-{ }_{\Delta} \mathrm{P}_{\mathrm{T}}$ (где ${ }_{\Delta} \mathrm{P}_{\mathrm{c}}-$ суммарные потери давления в трубопроводе с установленным регулирующим клапаном, ${ }_{\Delta} \mathrm{P}_{\mathrm{T}}-$ то же , на технологических аппаратах) .

Поскольку разность давлений равна сумме потерь давлений на участке, то согласно рис.2:

${ }_{\Delta} \mathrm{P}_{\mathrm{c}}=\mathrm{P}_{0}-\mathrm{P}_{\mathrm{n}} \pm \mathrm{Z}_{\gamma}$.

Где $Z_{\gamma}$ - гидростатический напор, создаваемый столбом жидкости высотой Z. Если принять гидравлическое сопротивление расчетного участка трубопровода (линии) без регулирующего клапана постоянным, то с увеличением расхода среды в регулируемой системе, т. е. с подъемом плунжера клапана и увеличением $K_{v}$, снижаются его гидравлическое сопротивление и перепад давлений на клапане. В то же время в связи с увеличением расхода увеличивается перепад давлений на линии. Поэтому ${ }_{\Delta} \mathrm{P}_{\min }={ }_{\Delta} \mathrm{P}_{\mathrm{c}}-{ }_{\Delta} \mathrm{P}_{\text {Tmax }}$.

Следовательно, с изменением расхода в системе изменяется отношение ${ }_{\Delta} \mathrm{P} /{ }_{\Delta} \mathrm{P}_{\mathrm{c}}$. С увеличением расхода в системе перепад давлений на клапане составляет все меньшую долю всего перепада давлений в систем

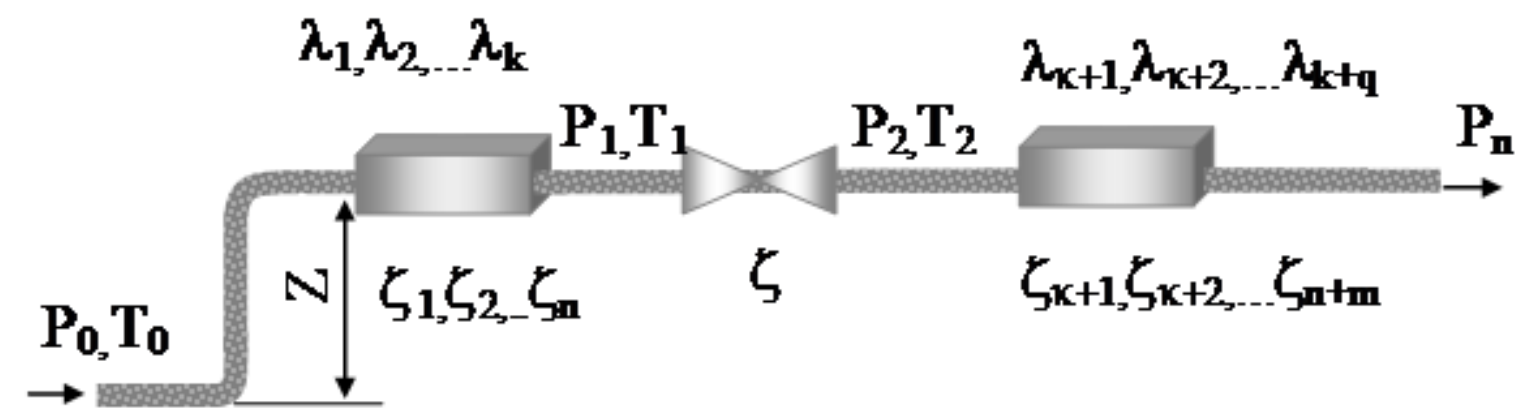

Рис. 2 - Расчетная схема участка трубопровода: $\mathrm{P}_{1}$ - абсолютное давление при минимальном рас ходе до исполнительного устройства; $\mathrm{P}_{2}$ - то же после исполнительного устройства; $\square_{1,}, \square_{2, \ldots} \square_{\mathrm{k}}-$ коэффициенты сопротовления трению на прямых участках трубопровода до исполнительного устройства; $\square \square \square 1, \square \square \square 2, \ldots \square$ k+q - то же после исполнительного устройства; $\square_{1}, \square_{2, . .} \square_{\mathrm{n}}-$ коэффициенты местных сопротивлений трубопровода до исполнительного устройства; $\square_{\square \square 1}, \square_{\square \square 2, \ldots} \square_{\mathrm{n}+\mathrm{m}}-$ то же после исполнительного устройства; $\mathrm{Z}$ - разность уровней верхней и нижней отметок трубопровода; $\mathrm{T}_{0}, \mathrm{~T}_{1}, \mathrm{~T}_{2}$ - абсолютные температуры газовой среды соответственно в начале участка, до исполнительного устройства и после него.

В этих условиях расход среды через систему изменяется не в соответствии с пропускной характеристикой клапана, а имеет отличный от нее характер, т.е. расходная характеристика клапана не совпадает с пропускной характеристикой. По существу, расходная характеристика клапана определяет собой расходную характеристику системы (с становленным на ней регулирующим клапаном), выражающую зависимость пропускной способности системы от подъема рабочего органа (плунжера) клапана[8]. Характер и величина различий между пропускной и расходной характеристиками определяются в зависимости от отношения,$n=K_{v_{y}} / K_{v_{T}}$.

Цель работы является поиск оптимальной системы управления шаровым краном на отдельном участке трубопроводного транспорта подачи сока (на базе привода BOXSL-R-03 КАМОЦЦИ - электропневмопозиционер ЭПП ) при соблюдении условия максимально точного совпадения требуемого и действительного значений перемещения рабочего звена, вала поворотного привода (с учетом необходимого запаса по расходу продукта).

При значении меньшем, чем требуется, не будет обеспечен максимальный расход среды через систему, при большем значении регулирующий клапан будет работать в более узком интервале значений $\mathrm{S}$, что ухудшает его эксплуатационные показатели: увеличивается погрешность регулирования, усиливается износ седла и плунжера в связи с работой на узких щелях и т. д. Поскольку ограниченная номенклатура выпускаемых регулирующих клапанов используется в разнообразных условиях эксплуатации, во многих случаях работа регулирующего клапана протекает в пределах части полного хода плунжера, определяемого рабочими значениями $K_{v_{\max }}$ $K_{v_{\min }}$ при соответствующих им рабочих значениях хода плунжера $S_{\max }$ и $S_{\min }$ определяемых по расходной характеристике. Значения $K_{v_{\max }}$ и $K_{v_{\min }}$ определяют собой рабочий участок пропускной характеристики клапана, 


\section{АВТОМАТИЧНІ ТА АВТОМАТИЗОВАНІ СИСТЕМИ УПРАВЛІННЯ ТЕХНОЛОГІЧНИМИ ПРОЦЕСАМИ}

$S_{\min }$ - рабочий участок хода плунжера. Это можно выразить и в относительных величинах: рабочий участок пропускной характеристики; $l_{\max }-l_{\min }-$ рабочий участок $q_{\max }-q_{\min }$ хода плунжера.

ЭПП конструктивно представляет собой алюминиевый корпус в котором находится пневматические распределители, контроллер управления и угловой датчик положения (рис.3).

Работает ЭПП следующим образом. На контроллер поступает сигнал управления 4-20мА. Этот сигнал сравнивается с сигналом датчика положения. При отличии сигнала датчика от сигнала управления подаётся сигнал на соответствующий соленоид пневмораспределителя, подавая сжатый воздух на соответствующий выход до тех пор, пока сигналы не уравняются. Подключение внешних цепей питания и сигнала управления производится к клемной колодке, расположенной внутри корпуса ЭПП[1].

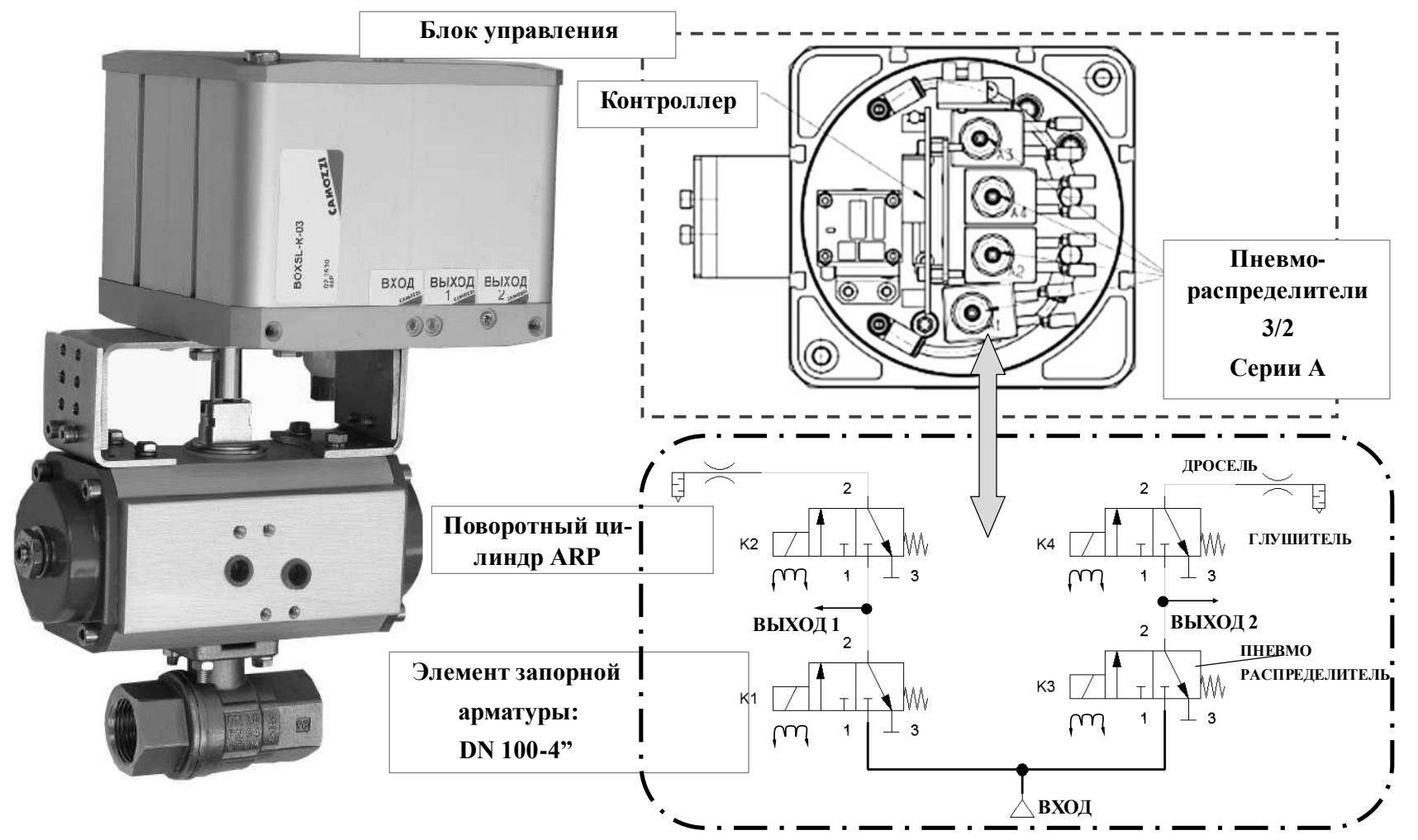

Рис.3 - Принципиальная схема электропневматического позиционера с внешним подключением элемента запорной арматуры (шарового крана DN $100-4$ ")

Алгоритм работы контроллера определяелся программой зашитой в память микропроцессора контроллера.

Винтовые терминалы ЭПП были использованы: ХT1 - для подключения внутренних цепей, (датчик, катушки пневмоклапанов); ХT2 - для подключения внешних цепей, (питание, линии управления 4-20ма, линии обратной связи). Группа переключателей SW1 выполняла функцию установки режимов работы и необходимых функций и параметров, определяемых условиями работы трубопроводной магистрали. Также, при проведении эксперимента была использована светодиодная индикация: L1(красный) и L2(жёлтый) предназначены для индикации состояния дискретных выходов, ошибок работы в рабочем режиме и для вспомогательной индикации в остальных режимах работы контроллера. Контроллер управлял сигналами на дискретных выходах, в соответствии с управляющим сигналом и установленной программой.

Для подключения ЭПП, также была задействованна (по специальному алгоритму (1)) функция автокалибровки. Данная функция позволяет программно нормировать функцию преобразования датчика и сигнал обратной связи, выдаваемый контроллером. Особенно это важно в ситуации, когда рабочий диапазон датчика больше диапазона перемещения вала привода или механизма запорной арматуры, которой он управляет.

При этом контроллер вычисляет и запоминает коэффициенты для нормирования функции преобразования датчика и сигнала обратной связи, выдаваемого контроллером. Следящий привод переходит в рабочий режим, и теперь позиционирование осуществляется во всем диапазоне поворота вала, при изменении сигнала управления от 
4мА до 20мА. Соответственно и сигнал обратной связи меняется от 4мА до 20мА во всём диапазоне позиционирования.

a)

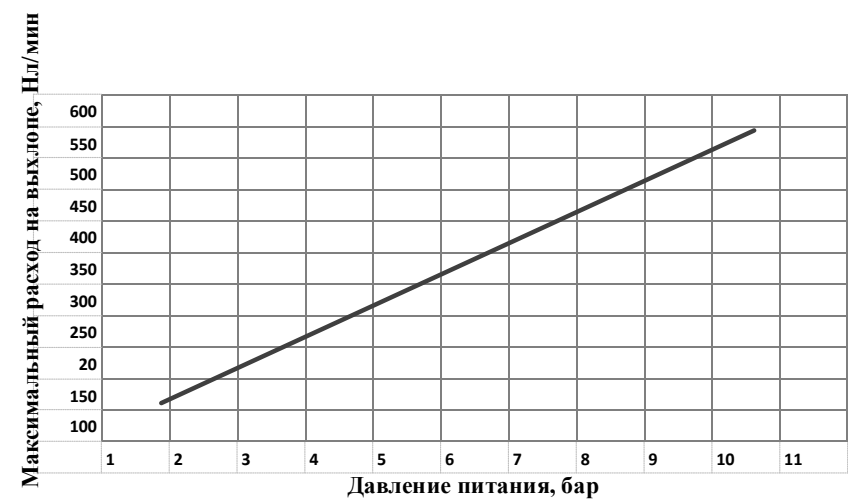

б)

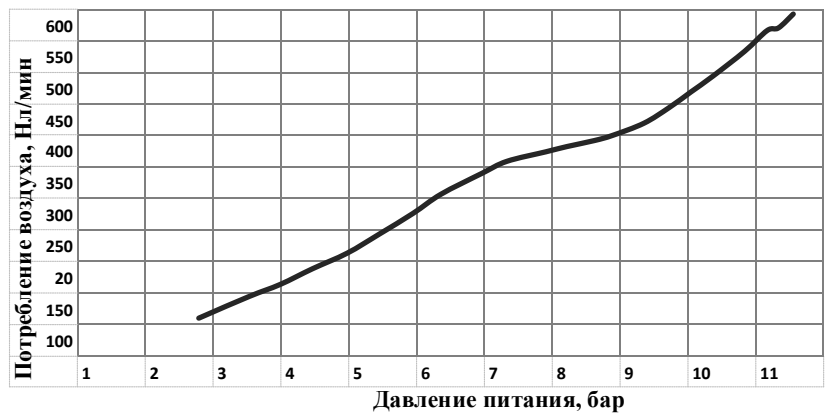

Рис. 4 - Исследование изменения расходных характеристик ЭПП от изменения рабочего давления пневмомагистрали: а) максимальный расход на выхлопе; б) потребление при неподвижном объекте (в режиме позиционирования)

Принцип построения системы управления аналогичен рассмотренным линейным приводам [2], при этом выбранная модификация BOXSL-R-03 позволяет напрямую, без передаточных механизмов, осуществлять управление запорными элементами (в том числе и шаровыми кранами). Полученные результаты расходных характеристик (рис.4), позволяют правильно подобрать ЭПП по требуемому расходу основного енергорессурса(сжатого воздуха) - учитывая особености работы управляемого им элемента.

\section{Выводы}

При тестировании работы ЭПП в системе с трубопроводной арматурой, погрешность позиционирования привода(рабочего вала поворотного цилиндра ARP) составила не более $1^{\mathrm{O}}$.

В конечном итоге, выбор регулирующего клапана из числа серийно выпускаемых по его гидравлическим параметрам сводится к выбору вида пропускной характеристики (линейная или равнопроцентная) и его условного диаметра прохода Dy в мм. Условный диаметр прохода Dy регулирующего клапана определяется по требуемой величине $K_{v_{y}}$, которая находится из условия, где $K_{v_{\max }}$ - наибольшее рабочее (требуемое расчетное) значение $K_{v}$ при полном повороте крана (открытое сечение). В реальных условиях эксплуатации трубопроводных систем перепад давлений на регулирующем клапане не остается постоянным, а изменяется в зависимости от гидравлических характеристик насосной установки, составляющих элементов трубопроводной системы, расхода среды потребителями, свойств перемещаемой среды, ее вязкости, гидравлического режима движения, способности вскипания в связи с понижением давления и некоторых других факторов. Пропускная характеристика должна быть выбрана так, чтобы в эксплуатационных условиях создавалась требуемая расходная характеристика.

\section{Литература}

[1] Технічні інформаційні ресурси. [Електронний ресурс] : [Веб-сайт]. - Електронні дані. - Київ :CAMOZZI. Режим доступу до каталогу.: http://catalog.camozzi.ua - Назва з екрану;

[2] Повышение уровня технологической эффективности трубопроводной арматуры позиционным электропневмоприводом // Ж-л «Автоматизація технологічних та бізнес-процесів». - Одесса:ОНАХТ т.№7(1)/2015.- С.68-74;

[3] А.И.Гошко Арматура трубопроводная целевого назначения/ Москва.- Машиностроение.- 2003.- 280с;

[4] Концепции повышения эффективности АСУ ТП при производстве сахара в Украине / С.А. Ляшенко, А.С. Ляшенко, И.С. Беляева // Сучасні напрямки технології та механізації процесів переробних і харчових виробництв. Вісник ХНТУСГ. - Харків: ХНТУСГ, 2008. - Вип. 74. - С. 54-63;

[5] Стратегия автоматизации производства сахара / Белоусов В.Ю., Литвинов А.Ф.. Потапов О.А., Горчинский Ю.H. // Caxap. -2002.-№1.- С. 28-33;

[6] Сапронов А.Р. Технология сахарного производства / Сапронов А.Р. -М.: Агропромиздат, 1986. - 436c; 
[7] Буряковий цукор технології виробництва / M.І. Бахмат, М.I. Ігнатьев, І.А. Вітвіцький. - Кам'янець Подільський: Абетка - НОВА. 2004.-372c;

[8] Системы автоматизации технологических процессов сахарного производства / О.Яковлев, С. Танцюра, А. Войтюк, Ю. Рудаков, С. Латышев, В. Волков, М. Рак, Н. Круглый // Пищевая промышленность. 2000 - №1. - C.44-53.

References

[1] Tehnichni informatsiyni resursi. [Elektronniy resurs] : [Veb-sayt]. - Elektronni dani. - Kiyiv :CAMOZZI. Rezhim dostupu do katalogu.: http://catalog.camozzi.ua - Nazva z ekranu;

[2] Povyshenie urovnja tehnologicheskoj jeffektivnosti truboprovodnoj armatury pozicionnym jelektropnevmoprivodom // Zh-1 «Avtomatizacija tehnologichnih ta biznes-procesiv». - Odessa:ONAHT t.№7(1)/2015.- S.68-74;

[3] A.I.Goshko Armatura truboprovodnaja celevogo naznachenija/ Moskva.- Mashinostroenie.- 2003.- 280s;

[4] Koncepcii povyshenija jeffektivnosti ASU TP pri proizvodstve sahara v Ukraine / S.A. Ljashenko, A.S. Ljashenko, I.S. Beljaeva // Suchasni naprjamki tehnologiï ta mehanizaciï procesiv pererobnih $\mathrm{i}$ harchovih virobnictv. Visnik HNTUSG. - Harkiv: HNTUSG, 2008. - Vip. 74. - S. 54-63;

[5] Strategija avtomatizacii proizvodstva sahara / Belousov V.Ju., Litvinov A.F.. Potapov O.A., Gorchinskij Ju.N. // Sahar. -2002.-№1.- S. 28-33;

[6] Sapronov A.R. Tehnologija saharnogo proizvodstva / Sapronov A.R. -M.: Agropromizdat, 1986. - 436s.

[7] Burjakovij cukor tehnologiï virobnictva / M.I. Bahmat, M.I. Ignat'ev, I.A. Vitvic'kij. - Kam'janec' - Podil's'kij: Abetka - NOVA. 2004.-372s;

[8] Sistemy avtomatizacii tehnologicheskih processov saharnogo proizvodstva / O.Jakovlev, S. Tancjura, A. Vojtjuk, Ju. Rudakov, S. Latyshev, V. Volkov, M. Rak, N. Kruglyj // Pishhevaja promyshlennost'. 2000 - №1. - S.44-53.

\title{
СИСТЕМА АВТОМАТИЧЕСКОГО РЕГУЛИРОВАНИЯ РАСХОДА СЫПУЧЕГО МАТЕРИАЛА
}

The system automatically controlling the flow of bulk material

Павлов А.И. ${ }^{1}$

${ }^{1}$ Одесская национальная академия пищевых технологий, г. Одесса

Copyright (C 2014 by author and the journal "Automation technological and business - processes". This work is licensed under the Creative Commons Attribution International License (CC BY). http://creativecommons.org/licenses/by/4.0/

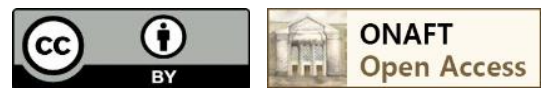

DOI: $10.15673 /$

\begin{abstract}
Аннотация
Задача управления расходом сыпучих материалов является весьма актуальной при автоматизации производственных процессов, связанных с необходимость измельчения, смешивания, растворения, сушки, обжига различных компонентов. Использование в системах автоматического регулирования разнообразных питателей приводит к большим финансовым затратам как на стадии их создания, так и при эксплуатации. Альтернативным решением задачи регулирования расхода сыпучих материалов является использование в качестве регулирующего органа подбункерных задвижек. При этом не требуется измерять величину расхода сыпучего материала. В качестве регулируемой координаты используется косвенный параметр величина открытия задвижки. Необходимая величина открытия задвижки для обеспечения заданного
\end{abstract}

